\title{
Celiac disease: pathogenesis of a model immunogenetic disease
}

\author{
Martin F. Kagnoff \\ Departments of Medicine and Pediatrics, Laboratory of Mucosal Immunology, and Wm. K. Warren \\ Medical Research Center for Celiac Disease, UCSD, La Jolla, California, USA.
}

\begin{abstract}
Celiac disease is characterized by small-intestinal mucosal injury and nutrient malabsorption in genetically susceptible individuals in response to the dietary ingestion of wheat gluten and similar proteins in barley and rye. Disease pathogenesis involves interactions among environmental, genetic, and immunological factors. Although celiac disease is predicted by screening studies to affect approximately $1 \%$ of the population of the United States and is seen both in children and in adults, $10 \%-15 \%$ or fewer of these individuals have been diagnosed and treated. This article focuses on the role of adaptive and innate immune mechanisms in the pathogenesis of celiac disease and how current concepts of immunopathogenesis might provide alternative approaches for treating celiac disease.
\end{abstract}

Celiac disease (CD) is characterized by small-intestinal mucosal injury and nutrient malabsorption. It is activated in genetically susceptible individuals by the dietary ingestion of proline- and glutamine-rich proteins that are found in wheat, rye, and barley and are widely termed "gluten" (1). Although approximately $1 \%$ of the population of the United States is affected by CD, most affected individuals remain undiagnosed. This probably reflects the fact that patients with $C D$ can manifest a spectrum of intestinal and/or extraintestinal symptoms and, in some cases, they can be relatively asymptomatic, with their disease first being detected by antibody screening because they were identified as being at high risk of developing CD (for example, by being a family member of an affected patient) (2). Presumed disease is best detected by serologic screening for the presence of IgA antibodies specific for tissue TGase, and this should be followed by biopsy of the mucosa of the small intestine to establish a definite diagnosis (3). Lifethreatening complications, although relatively rare, can include the development of refractory $\mathrm{CD}$ and enteropathy-associated $\mathrm{T}$ cell lymphomas (EATLs) (4-6).

As is discussed in this article, acquired T cell-mediated immune mechanisms and innate immune mechanisms have an important role in the pathogenesis of CD (1). Although most individuals respond to treatment with a "gluten"-free diet (GFD), which is the only currently accepted therapy, recent advances in our understanding of the immunopathogenesis of CD might lead to alternative treatments for this disease.

\section{Does disease pathology provide clues to disease pathogenesis?}

In considering the immunopathogenesis of $\mathrm{CD}$, it can be helpful to look at the wide spectrum of pathology seen in this disease. When viewed through a dissecting microscope, the luminal surface of a small-intestinal mucosal biopsy from a healthy subject has abundant villi (Figure 1A) (reminiscent of the shag household carpets of our parents' generation!). In contrast, the luminal surface of a small-intestinal mucosal biopsy from a patient with

Nonstandard abbreviations used: CD, celiac disease; EATL, enteropathy-associated T cell lymphoma; GFD, "gluten"-free diet; IEL, intraepithelial lymphocyte.

Conflict of interest: The author has declared that no conflict of interest exists. Citation for this article: J. Clin. Invest. 117:41-49 (2007). doi:10.1172/JCI30253. severe CD (Figure 1B) manifests a complete loss of villi, with a flat mucosal surface accentuated by ridges and numerous crypt openings. When tissue sections of the mucosa of the small intestine are stained with $\mathrm{H} \& \mathrm{E}$, to visualize mucosal structure and the individual cells, the mucosa of healthy individuals is characterized by tall villi lined by a single layer of columnar epithelial cells with nuclei located near the basal surface; a smattering of intraepithelial lymphocytes (IELs) (approximately 1 per 6-10 epithelial cells); lymphocytes and plasma cells in the lamina propria in numbers consistent with the "physiologic inflammation" that is normal in the small intestine; and a ratio of villous height to crypt depth of approximately 4:1 to 5:1 (Figure 1C). In contrast, the small-intestinal mucosa of patients with severe CD shows total villous atrophy (Figure 1D). The complete loss of villi is accompanied by the presence of markedly abnormal squamoid surface epithelial cells, an increase in the number of IELs, a marked increase in the number of lymphocytes and plasma cells in the lamina propria, and striking crypt hypertrophy with increased crypt mitoses. Pathologic changes in less severe CD are not as marked and can be characterized by increased numbers of IELs and less extensive villous atrophy and crypt hypertrophy, which is termed subtotal villous atrophy. In addition to the high variability in the pathologic changes, clinical presentations also vary markedly. Indeed, only a small number of patients present with the "classical" symptoms of marked weight loss, malnutrition, and steatorrhea. In contrast, many individuals with CD manifest predominantly extraintestinal symptoms and findings (for example, unexplained iron deficiency anemia, premature-onset osteoporosis, irritability, and depression) or are relatively asymptomatic (for example, individuals identified only because they have affected family members). CD has many characteristics of a chronic inflammatory disease. Consistent with this, a substantial mucosal infiltration of neutrophils, the hallmark of an acute inflammatory response, is not seen in biopsies from either mild or more severe disease.

\section{Immunopathogenesis of CD}

Susceptibility to CD, and its activation and perpetuation, involve a combination of environmental and genetic factors, and immunological mechanisms. As I discuss here, a number of important factors and mechanisms underlying disease pathogenesis are well defined, whereas others are only now coming into focus. 
A

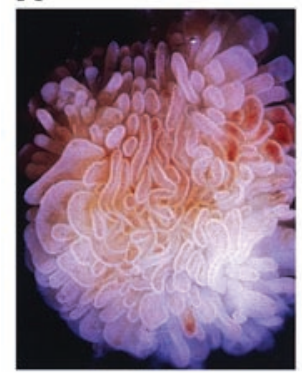

B

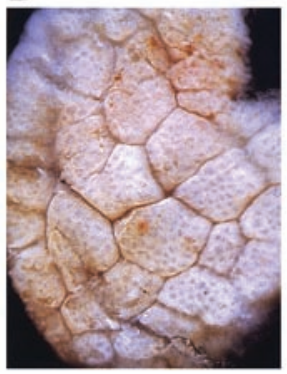

C

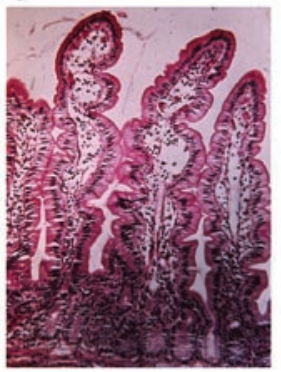

D

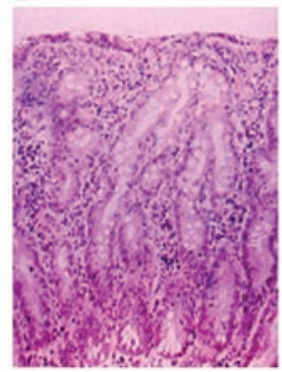

Figure 1

Small-intestinal mucosal biopsy. (A and B) Small-intestinal mucosal biopsy viewed through a dissecting microscope. The normal biopsy (A) shows numerous surface villi, whereas a biopsy from an individual with $C D$ and total villous atrophy shows, in place of the villi, numerous surface openings to underlying crypts and surface ridges (B). (C) H\&E-stained section of a normal small-intestinal mucosal biopsy. Original magnification, $\times 400$. (D) H\&E-stained section of a small-intestinal mucosal biopsy from an individual with CD and total villous atrophy. Original magnification, $\times 400$. All panels reprinted with from Gastroenterology $(1)$ with permission from the American Gastroenterological Association.

The role of dietary proteins in disease pathogenesis. $\mathrm{CD}$ is activated by proteins in the dietary cereal grains wheat, rye, and barley. Although the disease-activating proteins in these grains are widely termed "gluten" (and for simplicity are referred to here in quotation marks), strictly speaking, gluten only encompasses the diseaseactivating proteins in wheat. Gluten includes 2 major protein types, the gliadins and glutenins, both of which contain disease-activating peptides (7-9). The closely related proteins in barley and rye that activate $\mathrm{CD}$ are the hordeins and secalins, respectively $(10-12)$. Wheat, rye, and barley have a common ancestral origin in the grass family. Oats are thought to activate CD only rarely (13-15), and, consistent with this, oat avenins are more distantly related to the analogous proteins in wheat, rye, and barley and have a substantially lower proline content. The analogous proteins in rice, maize, sorghum, millet, Job's tears, and tef are still more distantly related and do not activate CD (Figure 2) (10, 11, 16).

Gliadins, glutenins, hordeins, and secalins have a high proline and glutamine content. The high proline content renders these proteins resistant to complete proteolytic digestion by gastric, proteins. However, failure to digest these and other proteins might be exaggerated in the small intestine of individuals with active disease who manifest epithelial cell brush border injury and accompanying pancreatic dysfunction. Interestingly, prolyl endopeptidases produced by certain bacteria and fungi can digest these proline-rich "gluten" peptides, and treatments based on the use of such enzymes have been suggested as a possible therapeutic adjunct to the standard $\operatorname{GFD}(17,18,20)$.

Genetic factors: MHC class II HLA-DQ alleles. The pathogenesis of $\mathrm{CD}$ is firmly rooted in host genetic factors. This was first evident from clinical observations of multiple cases of CD within families, and the high (approximately 70\%-75\%) rate of concordance for $\mathrm{CD}$ among monozygotic twins (21). It is known that CD is associated with specific MHC class II alleles that map to the $H L A-D Q$ locus (22). Moreover, the presence of specific $H L A-D Q$ alleles is necessary, although not sufficient, for the phenotypic expression of CD in virtually all affected individuals, irrespective of geographic location (23). Indeed, as is illustrated in Figure 3, almost all individuals with biopsy-confirmed CD express

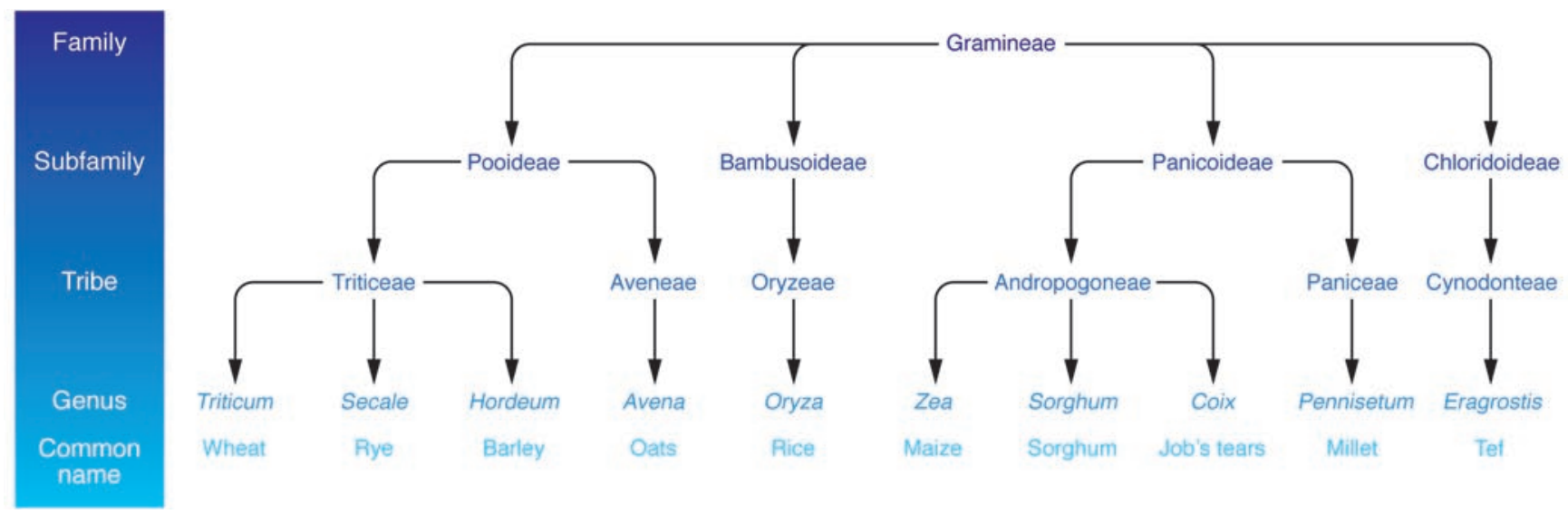

Figure 2

Taxonomy of some dietary grains. Wheat, barley, and rye, which contain the CD-activating proteins gluten, hordein, and secalin, respectively, are derived from the Triticeae tribe of the grass (Gramineae) family. In contrast, oats, which contain few CD-activating proteins, are more distantly related, as are rice, maize, sorghum, millet, Job's tears, and tef. 


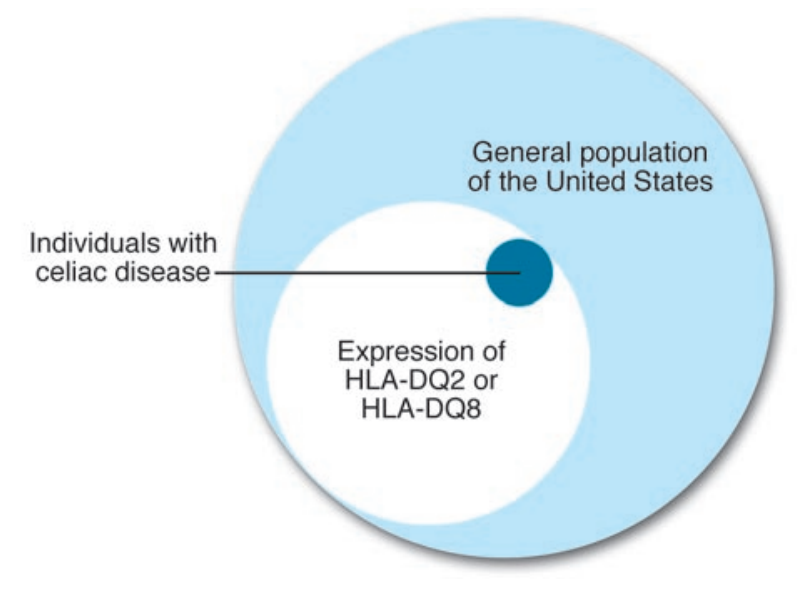

Figure 3

Venn diagram depicting the distribution of HLA-DQ2 and HLA-DQ8 in the general population and in CD. The MHC class II heterodimers HLA-DQ2 and HLA-DQ8 are commonly expressed by the general population of the United States. With few, if any, exceptions, patients with CD carry the HLA-DQ alleles $H L A-D Q B 1^{*} 02$ and $H L A-D Q A 1{ }^{*} 05$, which encode the HLA-DQ2 heterodimer, or $H L A-D Q B 1{ }^{*} 0302$ and $H L A-D Q A 1{ }^{*} 03$, which encode the HLA-DQ8 heterodimer.

$H L A-D Q$ alleles that encode specific HLA-DQ2 heterodimers or specific HLA-DQ8 heterodimers, and the alleles that encode these heterodimers are relatively common in the white population. The HLA-DQ2 heterodimers that confer susceptibility to CD are formed by a $\beta$ chain encoded by the allele $H L A-D Q B 1 * 02$ (either $H L A-D Q B 1 * 0201$ or $\left.{ }^{*} 0202\right)$ and an $\alpha$ chain encoded by the allele $H L A-D Q A 1 * 05$. This HLA-DQ2 heterodimer is present in aat least $90 \%-95 \%$ of patients with CD (22), although a very small number of CD patients have been reported in whom only 1 of these DQ2 alleles is present (that is, $H L A-D Q B 1 * 0202$ or, rarely, $H L A-D Q A 1 * 05$ ) (23). The HLA-DQ8 heterodimer found in the remaining $5 \%-10 \%$ of patients with CD is formed by the $\beta$ chain and $\alpha$ chain encoded by $H L A-D Q B 1 * 0302$ and $H L A-D Q A 1 * 03$, respectively $(24,25)$.

The HLA-DQ2 alleles associated with increased susceptibility to $\mathrm{CD}$ can be inherited in cis (that is, on 1 parental chromosome) or in trans, with the HLA-DQ alleles forming the HLA-DQ2 heterodimer being encoded on 1 chromosome from each parent (Figure 4) $(26,27)$. Notably, CD is substantially more prevalent in those in whom $100 \%$ or approximately $50 \%$ of the HLA-DQ heterodimers are HLA-DQ2 than in those in whom only approximately $25 \%$ of the HLA-DQ heterodimers are HLA-DQ2 (28-30). In this regard, the approximately $2 \%$ of the population who are homozygous for the HLA-DQ2 heterodimer account for approximately 25\% of all patients with CD. Notably, an increased abundance of HLA-DQ2 heterodimers on APCs has correlated with an increased magnitude of in vitro gluten-specific $\mathrm{T}$ cell responses (30), which, if paralleled in vivo, might contribute to the increased risk of developing clinically apparent CD in individuals homozygous for HLA-DQ2. Once CD develops, the clinical course seems generally similar whether or not $100 \%, 50 \%$, or $25 \%$ of the HLA-DQ molecules form the HLA-DQ2 heterodimer, although being homozygous for $H L A-D Q B 1 * 0201$ recently was noted to be associated with more severe disease (31). The CD-associated $H L A-D Q 2$ susceptibility alleles are very underrepresented in Japan, and, consistent with this, CD is extremely rare in Japan (only 2 reported cases) (32).
Since $H L A-D Q$ alleles account for only approximately $40 \%$ of the genetic susceptibility to $C D$, extensive efforts have been made to identify additional genes associated with $\mathrm{CD}$, most recently with the use of genome-wide screening approaches (33-43). Candidate genetic regions that possibly increase CD susceptibility have been noted in some populations on chromosomes 2,3 , 4, 5, 6 (telomeric of the HLA locus), 9, 11, 18, and 19 (33-43). However, the putative risk posed by these regions is substantially lower than that posed by expression of the HLA-DQ2 or HLA-DQ8 CD susceptibility alleles. Furthermore, the genes in these regions and the possible mechanisms by which they might contribute to disease susceptibility currently are not known, although some of these regions also have been associated with other autoimmune or inflammatory diseases. Recently, a variant of the gene encoding myosin IXB was proposed as an important CD risk factor in a Dutch cohort of patients with CD (44), but this has proven controversial, as marked increases in variants of the same gene were not found in either British or Norwegian/Swedish cohorts $(45,46)$. Although there are likely to be a number of different genes involved in CD susceptibility, it is possible that these vary in their contribution to disease susceptibility among different individuals, making them hard to identify.

Adaptive immunity: activation of HLA-DQ-restricted mucosal T cells and the role of tissue TGase. How do HLA-DQ2 and HLA-DQ8 contribute to the immunopathogenesis of CD? To answer this question, this article focuses on the HLA-DQ2 heterodimer encoded by $H L A-D Q B 1 * 02$ and $H L A-D Q A 1 * 05$, as most CD patients carry this heterodimer and much of the structural biochemistry completed to date has used this heterodimer as a model. Nonetheless, similar considerations, with some variation, probably apply also to the HLA-DQ8 heterodimer.

HLA-DQ2 and HLA-DQ8 heterodimers on APCs can bind and subsequently present "gluten" peptides to populations of $\mathrm{CD} 4^{+} \mathrm{T}$ cells in the lamina propria of the small intestine (25, 47-49). How HLA-DQ2 and HLA-DQ8 bind such peptides was an enigma for several years, because the peptide-binding groove of HLA-DQ2 and HLA-DQ8 favors the binding of peptides with negatively charged residues at key anchor positions. Such negatively charged amino acids are largely absent from native "gluten" peptides generated in the human intestinal tract. However, this puzzle was solved after the discovery that the target antigen of an autoantibody present in many patients with CD was a calcium-dependent tissue TGase (50).

Tissue TGase, which is released in the intestinal mucosa during tissue injury, has a role in tissue repair and cross-links proteins by forming isopeptide bonds between glutamine and lysine residues. However, tissue TGase also has a high avidity for "gluten" peptides and, under certain conditions (for example, low $\mathrm{pH}$ ) and in the absence of lysine residues, can deamidate glutamine (51,52), which converts neutral glutamine to negatively charged glutamic acid $(51,53,54)$. Further studies indicated that tissue TGase has specificity for only selected glutamine residues in the glutamine- and proline-rich "gluten" peptides, which depends on the amino acids neighboring the target glutamine residue. Some, but not all, of the deamidated "gluten" peptides, by virtue of having negatively charged glutamic acid residues, manifest an increased binding affinity for the disease-relevant HLA-DQ2 or HLA-DQ8 molecules (51, 52).

Once bound to HLA-DQ2 and HLA-DQ8, the "gluten"-peptideHLA-DQ complexes can activate T cells in the mucosa of the small intestine that recognize these complexes $(51,55)$. Importantly, large "gluten" peptides that contain multiple HLA-DQ binding 

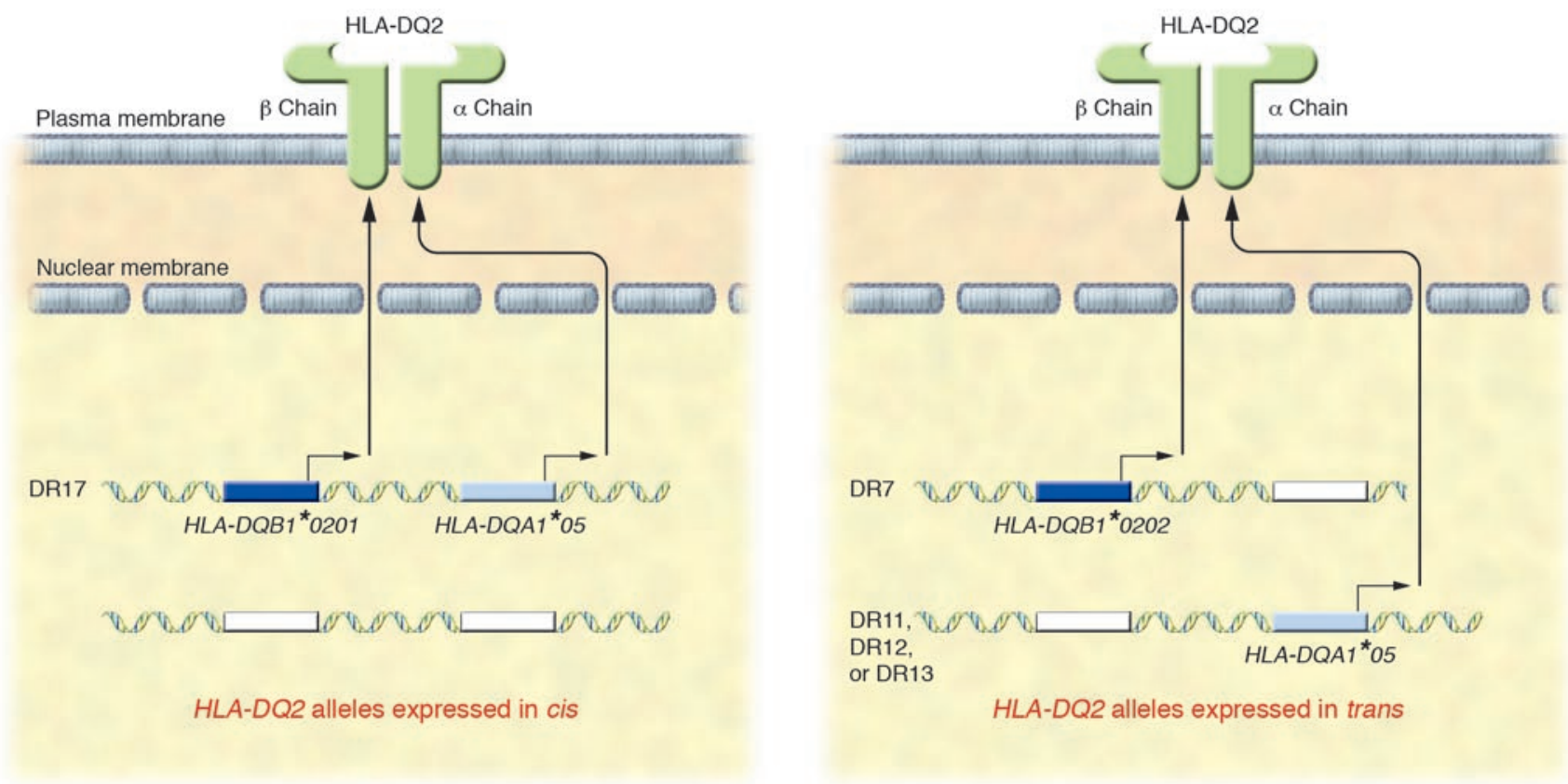

Figure 4

Two ways to inherit the HLA-DQ2 heterodimer associated with CD. DR17 haplotypes (formerly termed DR3) carry in cis (that is, on the same chromosome) the $H L A-D Q$ alleles $H L A-D Q B 1^{*} 0201$, which encodes a $\beta$ chain, and $H L A-D Q A 1 * 05$, which encodes an $\alpha$ chain. The $\beta$ and $\alpha$ chains form an HLA-DQ2 heterodimer that is associated with CD. DR7 haplotypes carry the very closely related $H L A-D Q B 1 * 0202$ allele on 1 chromosome. If the other chromosome carries a DR11, DR12, or DR13 (formerly termed DR5) haplotype that has the HLA-DQA1*05 allele, the $\beta$ and $\alpha$ chains encoded by those alleles can pair in the cell and form the CD-associated HLA-DQ2 heterodimer. If an individual is homozygous for DR17, or heterozygous for DR17/DR7, $100 \%$ or $50 \%$, respectively, of their HLA-DQ molecules are presumed to be the CD-associated HLA-DQ2 heterodimer.

epitopes $(56,57)$ have greater $\mathrm{T}$ cell-stimulatory activity than small peptides containing a single HLA-DQ2 binding sequence $(19,58)$. In this regard, a deamidated immunodominant 33 -amino acid peptide of an $\alpha$-gliadin can be recognized by $T$ cells isolated from the small intestine of a number of adult HLA-DQ2-positive patients with $\mathrm{CD}$, and it was suggested initially that $\mathrm{T}$ cell responses in HLA-DQ2-positive adult CD patients might be directed to a very limited number of deamidated "gluten" peptides $(56,59,60)$. Nonetheless, HLA-DQ2-restricted T cells from children with CD recognize many different "gluten" peptides. That can also include, in some cases, "gluten" peptides that do not contain deamidated glutamine residues; this indicates that glutamine deamidation is not an absolute requirement for $\mathrm{T}$ cell activation early in the course of disease in children (61). It is now known that $\mathrm{T}$ cells in adults with CD also are reactive to multiple peptides from $\alpha$ - and $\gamma$-gliadins. The fact that "gluten"-reactive T cells from the mucosa of the small intestine of patients with CD can recognize a broad repertoire of "gluten" peptides and that these peptides can differ from patient to patient, renders therapeutic approaches designed to remove $\mathrm{T}$ cell-stimulatory epitopes from disease-activating dietary grains somewhat challenging.

The production of IFN- $\gamma$ is a signature of "gluten" peptide-specific HLA-DQ2- and HLA-DQ8-restricted T cells that are isolated from the mucosa of the small intestine of CD patients, and it is considered to have a key role in the downstream initiation of mucosal damage (62). Neutralization of IFN- $\gamma$ has been shown to prevent "gluten"-induced mucosal damage, at least in biopsies of CD mucosa maintained in organ culture (63). The commitment of "gluten"-reactive $\mathrm{CD} 4^{+} \mathrm{T}$ cells from the mucosa of patients with
CD to produce IFN- $\gamma$ is consistent with reports that the transcription factor T-bet, which directs Th1 cell-lineage commitment, is upregulated in the mucosa of untreated CD patients and returns to normal levels after gluten is withdrawn from the diet (64). In contrast to diseases that are characterized by a concurrent increase in IFN- $\gamma$ and IL-12, the mucosa of patients with CD is striking for its lack of increased IL-12 production, and this is consistent with a lack of STAT4 upregulation in the intestinal mucosa of patients with CD (64). Cytokines other than IL-12 (for example, IL-23 and IL-27) might induce expression of T-bet in the mucosa of patients with $\mathrm{CD}(65)$. This raises questions regarding the possible role of these cytokines in CD pathogenesis. Notably, there is no clear evidence as to whether or not tissue TGase-specific antibody has an important role in the pathogenesis of tissue injury.

Why is disease limited to those with HLA-DQ2 or HLA-DQ8? Why CD is limited to individuals with HLA-DQ2 or HLA-DQ8 was clarified by studies of HLA-DQ2 and HLA-DQ8 interactions with "gluten" peptides that can activate host $\mathrm{CD} 4^{+} \mathrm{T}$ cells in the intestinal mucosa, and by $\mathrm{x}$-ray crystallography of an HLA-DQ2 molecule containing a deamidated gluten peptide in its peptide-binding groove (66-70). Both approaches provided insights into the specialized characteristics of HLA-DQ2 (and HLA-DQ8) that allow them to bind prolinerich "gluten" peptides that contain deamidated glutamine residues. One unique feature of HLA-DQ2 (and HLA-DQ8) molecules is the presence in their peptide-binding groove of several "pockets" that favor binding of negatively charged residues, such as those found in "gluten" peptides in which glutamine has been deamidated to glutamic acid. Furthermore, HLA-DQ2, like other MHC class II molecules, prefers to bind peptides with a left-handed polyproline 
II helical configuration, which is a characteristic of these "gluten" peptides (11). Although proline-rich peptides generally disrupt hydrogen bonds important for peptide binding to most MHC class II molecules, HLA-DQ2 is optimally suited for the binding of proline-rich "gluten" peptides that have deamidated glutamines at specific residues in the correct binding register, without disrupting key hydrogen bonds (66). These findings provide an underpinning as to why HLA-DQ2 and HLA-DQ8 have such an important role in the development and amplification of $\mathrm{CD} 4^{+} \mathrm{T}$ cell responses in $\mathrm{CD}$.

How large is the estimated repertoire of different disease-activating gluten peptides? The number of different peptides that potentially can be generated from the digestion of wheat, rye, and barley "glutens" in the human small intestine is enormous. Nonetheless, based on known amino acid sequences in wheat gluten, barley hordeins, and rye secalins, coupled with assumptions as to which glutamine residues are targets for deamidation by tissue TGase, the spacing of proline residues in those peptides, and the known preferred binding motifs of HLA-DQ2 and HLA-DQ8, algorithms very roughly estimated that there might be as few as 50 peptides in wheat, approximately 60 in rye, and fewer than 35 in barley that can effectively bind HLA-DQ2 or HLA-DQ8. Such peptides represent candidate sequences for activating HLA-DQ2- and HLA-DQ8-restricted CD4 ${ }^{+} \mathrm{T}$ cells in the intestinal mucosa of patients with $\mathrm{CD}(71)$. Avenins in oats, which have a lower proline content, are predicted to contain very few possible disease-activating sequences. This is compatible with clinical observations that the consumption of oats, at least in moderation, is not a major contributor to the activation of this disease $(14,15)$. Laboratory findings also indicate that few avenin sequences activate mucosal T cells from patients with CD $(12,13,55)$.

Why doesn't everyone with HLA-DQ2 or HLA-DQ8 get CD, and, conversely, why do only a small minority of individuals with HLA-DQ2 or HLA-DQ8 get $C D$ ? HLA-DQ2 and HLA-DQ8 are present in approximately $40 \%$ of the United States population, but simply having HLA-DQ2 or HLA-DQ8 and ingesting products made from wheat, rye, and barley does not cause CD. This raises a key question: What other predisposing host factors or environmental events are necessary for activation of CD? This remains an open question. One could postulate (as discussed earlier) that there might be additional genes in some people that subtly control the permeability of the mucosa of the small intestine and therefore the access of "gluten" peptides to the underlying mucosa; differences in the function of APCs; or qualitative and/or quantitative differences in host innate immune responses to environmental events that determine disease susceptibility. Clearly, there also are a myriad of other possibilities.

One clue to an important event, however, might come from the fact that $\mathrm{CD}^{+} \mathrm{T}$ cells from the intestinal mucosa of $\mathrm{CD}$ patients that respond to "gluten" peptides produce the Th1 cytokine IFN- $\gamma$, coupled with the finding that under normal conditions, "gluten"reactive $T$ cells are not found in the mucosa of the small intestine. Activation of $\mathrm{CD}^{+} \mathrm{T}$ cells specific for "gluten" peptides in the lamina propria clearly requires that those peptides be presented by APCs (probably DCs) that express HLA-DQ2 or HLA-DQ8. Although HLA-DQ2 and HLA-DQ8 are relatively poorly expressed in normal mucosa, their expression can be upregulated on APCs activated by IFNs. Therefore, it is quite probable that the propensity to develop CD, at least in a subset of individuals, depends on the host's encounter with "gluten" peptides at a time when there is ongoing intestinal inflammation that provokes IFN production, the activation of APCs, and a Th1 cell response. Mucosal infections, notably with enteric viruses, would seem to be prime candidates that could set the stage for this type of host response. In this regard, enteric viruses can alter intestinal permeability to "gluten" peptides and upregulate the production of type I and type II IFNs, leading to upregulation of HLA-DQ2 and HLA-DQ8 on DCs. This can be accompanied by tissue damage with increased release of tissue TGase and perhaps also an increased influx of "gluten"-specific T cells from the periphery (72). In this hypothetical scenario, enteric viruses (and perhaps other enteric pathogens) create an optimal milieu for the activation of the HLA-DQ2- and HLA-DQ8-restricted "gluten"-specific Th1 cell response when those peptides "innocently" enter the cellular and cytokine milieu of the mucosal microenvironment. Interestingly, the onset of CD during the course of treatment of other diseases with IFN- $\alpha$ (for example, infection with HCV) (73-75) could also be interpreted to support such a scenario.

Role of innate immunity and IELs in CD. Recent studies suggest that activation of the innate immune system is important in the pathogenesis of CD and in some of the complications of this disease, namely in refractory CD (that is, severe villous atrophy and malabsorption that either does not respond or no longer responds to a GFD) and in the development of EATLs. In particular, an increase in the number of IELs in the mucosa of the small intestine is a characteristic feature of $\mathrm{CD}$, and those cells are likely to be important for the ongoing pathogenesis of CD (76-79). Furthermore, the IEL population has a substantial role in refractory CD and in the development of EATLs $(5,80)$. Following activation, IELs from patients with CD change from being typical antigenspecific $\mathrm{T}$ cells to being NK-like cells able to mediate epithelial cell damage through the recognition of stress-induced molecules on intestinal epithelial cells (79). The cytokine IL-15 takes center stage in this process. Upregulation of IL-15 expression by epithelial cells and DCs in the lamina propria in CD seems to contribute to altered signaling properties of the CD8 ${ }^{+}$IEL population. IL-15 also induces increased expression on intestinal epithelial cells of epithelial cell surface ligands (for example, MIC) that are targets of the cytotoxic, TCR-independent NK-like cells (78, 81-83). IL-15 produced by DCs in the CD mucosa also might be important in the adaptive $\mathrm{T}$ cell response to "gluten."

Peptides are in the amino-terminus of an $\alpha$-gliadin (p31-49 and p31-43), which are not thought to bind to HLA-DQ2 or HLADQ8, has been reported to upregulate IL-15 production by intestinal epithelial cells, to increase IEL infiltration and epithelial cell apoptosis in a human intestinal mucosal organ culture model, and to cause epithelial damage when instilled into the human duodenum $(77,84)$. These findings raise another question: Why does "gluten" not induce similar responses and epithelial cell damage in everyone's small intestine? Furthermore, it is not known whether IEL responses, which are putatively activated by "gluten" peptides, precede the in vivo activation of lamina propria "gluten"-specific CD4 ${ }^{+} \mathrm{T}$ cell responses or are activated secondarily to that response. In either case, to the extent that IEL and CD4 ${ }^{+}$ $\mathrm{T}$ cell responses are largely dependent on encounter with dietary "gluten" peptides, withdrawal of "gluten" from the diet would be predicted to abrogate both, and this is the case.

\section{A model of CD immunopathogenesis}

A model that I have proposed to conceptualize the role of the adaptive $\mathrm{T}$ cell response in $\mathrm{CD}$ pathogenesis (Figure 5) divides pathogenesis into 3 phases: luminal and early mucosal events; 


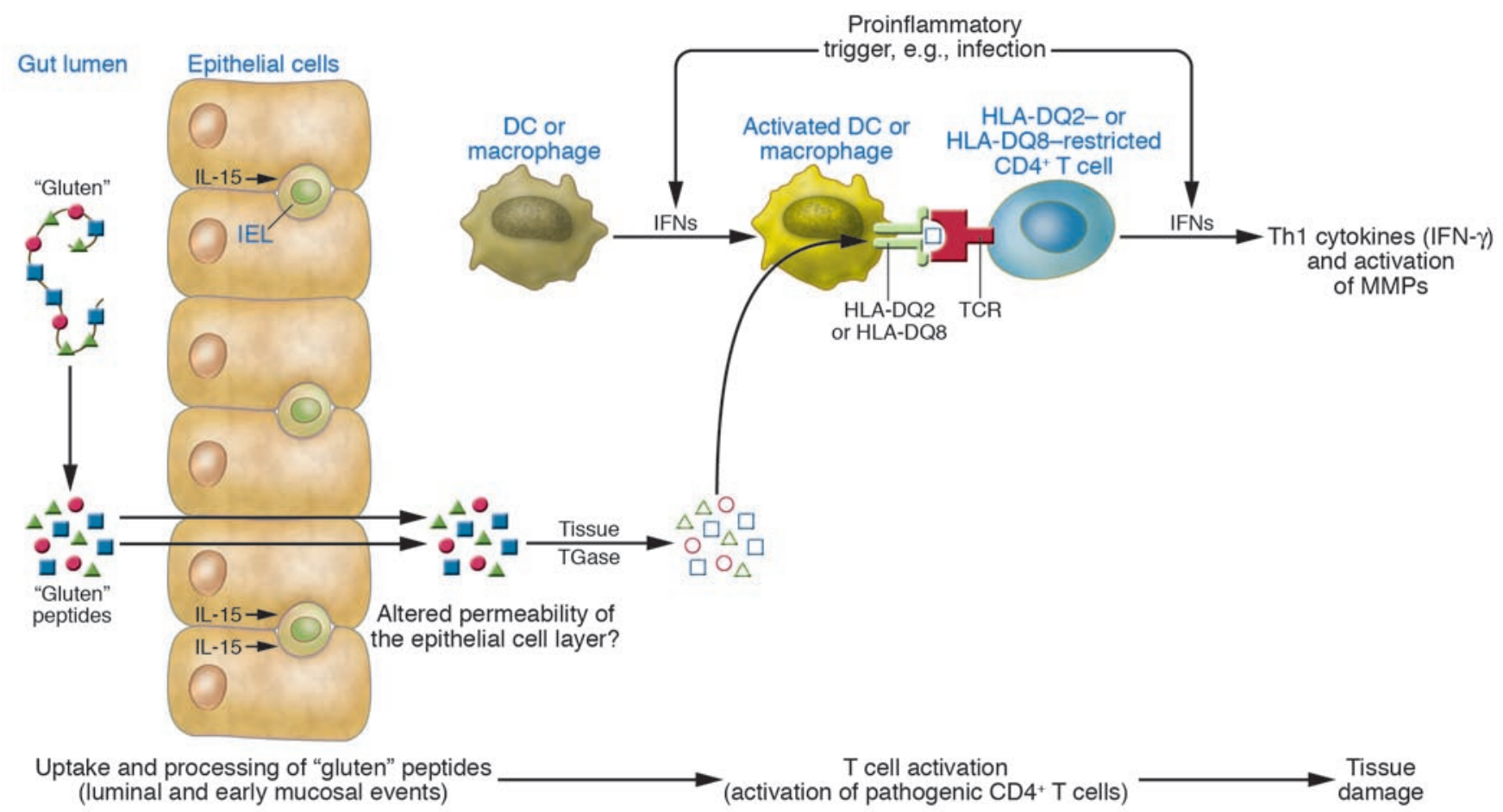

Figure 5

Pathogenesis of CD. This schematic divides the pathogenesis of CD into 3 major series of events: luminal and early mucosal events; the activation of pathogenic CD4+ T cells; and the subsequent events leading to tissue damage. During the luminal and early mucosal events, key features include the ingestion of "gluten" by a genetically susceptible individual. "Gluten" is not fully digested because of its high proline content, and this gives rise to a number of large undigested "gluten" peptides. The peptides cross the epithelial barrier to the lamina propria and encounter tissue TGase and APCs that express HLA-DQ2 or HLA-DQ8 heterodimers that are ideally suited to bind proline-rich peptides containing negatively charged glutamic acid residues as a result of glutamine deamidation by tissue TGase. In a further series of events, the APCs present some of these peptides to HLA-DQ2- and HLA-DQ8-restricted populations of CD4+ $T$ cells that become activated and release mediators that ultimately lead to tissue damage. There are still many unknowns. These include the mechanism by which "gluten" peptides cross the epithelial cell barrier, the role of innate immunity and IELs in both the early and the late phases of CD pathogenesis, the role of IL-15 and type I IFNs in disease pathogenesis, the underlying basis for the release of tissue TGase that leads to deamidation of gluten peptides, and the sequence of, and relationship between, CD4+ $\mathrm{T}$ cell responses and the responses of the IEL population.

activation of pathogenic $\mathrm{CD} 4^{+} \mathrm{T}$ cells; and events leading to tissue damage. In the first phase, an individual ingests "gluten." "Gluten" is digested to peptides, but because of the lack of prolyl endopeptidases among the gastric, pancreatic, and brush border enzymes, residual, relatively large "gluten" peptides that are rich in proline and glutamine remain after initial digestion. For 99\% of individuals, including most of those who carry the CD susceptibility alleles that encode $H L A-D Q 2$ and $H L A-D Q 8$, this does not present a problem, at least in terms of developing CD. However, in those HLADQ2- or HLA-DQ8-positive individuals with increased susceptibility to $\mathrm{CD}$, because of additional genetic and/or immunological factors and/or an adverse set of environmental events (for example, concurrent infection with an enteric virus), "gluten" peptides set in motion a series of immunological events that culminate in the immunopathology of CD.

Partially digested "gluten" peptides gain access to APCs in the subepithelial region of the small intestine, and the pathway(s) involved here is not yet determined but might include paracellular passage through a damaged epithelial cell layer, transepithelial passage, and/or the uptake of peptides by DC processes that can cross the epithelial cell layer (85). It is probable that the uptake of "gluten" peptides into a microenvironmental milieu in the small- intestinal mucosa suited for disease development is facilitated by a transient infection or other cause of inflammation in the small intestine. Viral infections would seem to be prime culprits to set the stage for a mucosal $\mathrm{T}$ cell response to "gluten" peptides. Having set the conditions for developing a Th1 cell response, in the second phase, "gluten" peptides bound to HLA-DQ2 or HLA-DQ8 encounter "gluten"-specific T cells that become committed to Th1 cytokine production. Activation of the "gluten"-specific CD4+ mucosal T cells is likely to be most pronounced in those individuals who are homozygous for HLA-DQ2 or in those who are heterozygous such that they have a double dose of the HLA-DQB1*02 allele. In the third phase, the release of IFN- $\gamma$ and other cytokines, which perpetuate the ongoing response and alter key mucosal functions including intestinal permeability, can also result in the activation and release of enzymes that can damage the mucosa, such as MMPs $(86,87)$. This results in a loss of villous structure and crypt hypertrophy. Although substantial advances have been made in understanding the role of adaptive $\mathrm{T}$ cell immunity in the pathogenesis of $\mathrm{CD}$, there nonetheless remain key holes in our knowledge and important open questions. For example, why is there an almost absolute skewing of the "gluten"-specific $\mathrm{CD}^{+}{ }^{+} \mathrm{T}$ cell response to HLA-DQrestricted $\mathrm{CD}^{+} \mathrm{T}$ cells in the intestinal mucosa, whereas the $\mathrm{CD}^{+}$ 
T cells that recognize "gluten" peptides in peripheral blood can also be HLA-DR restricted (88-90)? Furthermore, what is the relative importance of IFN- $\gamma$ produced by lamina propria CD4+ "gluten"specific T cells compared with IFN- $\gamma$ produced by the IEL population in CD? Importantly, why does one not see the classic CD lesion of crypt hypertrophy and villous atrophy in other mucosal inflammatory states that are also associated with increased IFN- $\gamma$ production (for example, Crohn disease)? It is important to ask also what role the balance between IFN- $\alpha$ and IFN- $\gamma$ production has in determining mucosal integrity and the outcome of cellular activation of NKG2 receptors on IEL.

How does one integrate newer findings with respect to the role of innate immunity into this disease model? It has been proposed that at least one $\alpha$-gliadin peptide that is not known to bind HLA-DQ2 or HLA-DQ8 can activate IL-15 production by intestinal epithelial cells. IL-15 in turn activates and leads to altered signaling through NKG2D and other CD94/NKG2 receptors on IELs whose cognate ligands on intestinal epithelial cells are upregulated by IL-15 and IFN- $\gamma$. This culminates in cytotoxic damage to the epithelium. Why "gluten" peptides that lack HLA-DQ2- or HLA-DQ8binding properties do not seem to activate such processes in all individuals is not known. Clearly the circumstances in vivo that govern the activation of this process are not well understood, and it is not known whether such events precede or follow activation of the "gluten"-specific adaptive T cell response. However, the fact that both the adaptive and the innate responses abate on a GFD suggests ways to probe their possible interdependence.

Does knowledge of the immunopathogenesis of CD suggest alternative or adjunctive therapentic approaches? A GFD currently is the only accepted therapy for CD. It has few risks and in most individuals is highly effective. Nonetheless, mainly for lifestyle and social reasons, many $\mathrm{CD}$ patients have a strong desire for alternative therapies. Recent insights into the immunopathogenesis of CD suggest potential alternative therapies to the GFD. However, consideration of alternative therapies must first take into account that, although inconvenient in several respects, the GFD is safe and effective. Therefore, one must carefully weigh the risks, benefits, and costs of alternatives, and carefully define under what conditions and indications such alternative therapies might be warranted.

Based on the central importance of HLA-DQ2 and HLA-DQ8 for the development of $\mathrm{CD}$, methods that attempt to block the binding of disease-activating "gluten" peptides to HLA-DQ2 or HLA-DQ8 seem an obvious approach (91). Nonetheless, potential pitfalls exist in the design of and the means of administering such "blockers." Furthermore, their possible effect on other host immune responses and their effectiveness in treating this disease are not known. Alternatively, approaches to block tissue TGase activity and the consequent deamidation of glutamine residues, which renders them better HLA-DQ2 or HLA-DQ8 binders, seem self-evident. However, whether inhibition of tissue TGase activity would be therapeutically useful is an open question, and a possible downside to such therapy could be inhibition of the important role that tissue TGase has in healing mucosal wounds. Further approaches to selectively delete specific $\mathrm{T}$ cell populations could also be envisioned, but here too risk/benefit and cost considerations could prove paramount.

Another alternative therapeutic approach could be to generate wheat varieties that lack or are markedly deficient in amino acid sequences that bind HLA-DQ2 or HLA-DQ8. At least 1 attempt to generate bread wheat devoid of disease-activating properties failed in the past (92) because of the high abundance of genes that encode gluten in the hexaploid genome of common bread wheat varieties. Therefore, it is difficult to delete a sufficient number of genes encoding the disease-activating gluten proteins but still retain the desirable baking qualities of wheat. Recent approaches to this problem have entailed searches for naturally occurring wheat varieties that, at the outset, have fewer $T$ cell-stimulatory sequences than common bread wheats $(93,94)$. However, since $\mathrm{T}$ cells from each individual with $\mathrm{CD}$ can respond to multiple different gluten peptides, and the array of $\mathrm{T}$ cell specificities for gluten peptides differs from person to person, it is not clear whether such approaches will yield therapeutically useful products devoid of disease-activating properties in most CD patients. Marked attention is being focused also on the development of enzymes taken orally that can digest proline- and glutamine-rich "gluten" peptides before they encounter disease-activating APCs in the mucosa of the small intestine (95-99). Finally, recent reports demonstrating the marked upregulation of IL-15 production by epithelial cells and DCs in the intestinal mucosa suggest that approaches to block IL-15 might abrogate disease activity. This could be a useful adjunct therapy, especially in the treatment of refractory $\mathrm{CD}$, where IL-15 seems to have a particularly important role through its activity on the IEL population.

\section{Conclusions}

In conclusion, some key aspects of the immunopathogenesis of $\mathrm{CD}$ and the genetic factors that govern host susceptibility to this disease are now well understood. Conversely, there are a number of important open questions and "missing links" that impede the full explanation of the pathogenesis of this disease. To explore a number of these important remaining questions, it seems essential to develop genetically manipulated animal models that recapitulate key events in the immunopathogenesis of this disease. Discovery and understanding of these missing links has great potential to lead to new approaches for the prevention, diagnosis, and treatment of CD. On a more general note, studies designed to unravel the pathogenesis of CD hold substantial promise for our understanding not only of this important gastrointestinal disease, but also of key mechanisms in other important autoimmune and inflammatory diseases.

Address correspondence to: Martin F. Kagnoff, Wm. K. Warren Medical Research Center for Celiac Disease, Room 412 MTF Building, UCSD, 9500 Gilman Drive, La Jolla, California 920930623, USA. Phone: (858) 534-4622; Fax: (858) 534-5691; E-mail: mkagnoff@ucsd.edu.

\footnotetext{
1. Kagnoff, M.F. 2005. Overview and pathogenesis of celiac disease. Gastroenterology. 128(Suppl. 1): S10-S18.

2. Green, P.H. 2005. The many faces of celiac disease: clinical presentation of celiac disease in the adult population. Gastroenterology. 128(Suppl. 1):S74-S78.

3. Rostom, A., et al. 2005. The diagnostic accuracy of serologic tests for celiac disease: a systematic review. Gastroenterology. 128(Suppl. 1):S38-S46.
}

4. Patey-Mariaud De Serre, N., et al. 2000. Distinction between coeliac disease and refractory sprue: a simple immunohistochemical method. Histopathology. 37:70-77.

5. Cellier, C., et al. 2000. Refractory sprue, coeliac disease, and enteropathy-associated T-cell lymphoma. French Coeliac Disease Study Group. Lancet. 356:203-208.

6. Catassi, C., Bearzi, I., and Holmes, G.K. 2005. Asso- ciation of celiac disease and intestinal lymphomas and other cancers. Gastroenterology. 128(Suppl. 1): S79-S86.

7. Van de Wal, Y., et al. 1999. Glutenin is involved in the gluten-driven mucosal T cell response. Eur. J. Immunol. 29:3133-3139.

8. Molberg, O., et al. 2003. Intestinal T-cell responses to high-molecular-weight glutenins in celiac disease. Gastroenterology. 125:337-344. 
9. Dewar, D.H., et al. 2006. The toxicity of high molecular weight glutenin subunits of wheat to patients with coeliac disease. Eur. J. Gastroenterol. Hepatol. 18:483-491.

10. Shewry, P.R., Tatham, A.S., and Kasarda, D.D. 1992. Cereal proteins and coeliac disease. In Coeliac disease. M.N. Marsh, editor. Blackwell Scientific Publications. London, United Kingdom. 305-342.

11. Kasarda, D.D. 1997. Gluten and gliadin: precipitating factors in coeliac disease. In Coeliac disease: proceedings of the 7th International Symposium on Coeliac Disease. September 5-7, 1996. Tampere, Finland. M. Maki, P. Collin, and J.K. Visakorpi, editors. Coeliac Disease Study Group. Tampere, Finland. 195-212.

12. Vader, L.W., et al. 2003. Characterization of cereal toxicity for celiac disease patients based on protein homology in grains. Gastroenterology. 125:1105-1113.

13. Arentz-Hansen, H., et al. 2004. The molecular basis for oat intolerance in patients with celiac disease. PLoS Med. 1:e1.

14. Janatuinen, E.K., et al. 2002. No harm from five year ingestion of oats in coeliac disease. Gut. 50:332-335.

15. Hogberg, L., et al. 2004. Oats to children with newly diagnosed coeliac disease: a randomised double blind study. Gut. 53:649-654.

16. Spaenij-Dekking, L., Kooy-Winkelaar, Y., and Koning, F. 2005. The Ethiopian cereal tef in celiac disease. N. Engl. J. Med. 353:1748-1749.

17. Hausch, F., Shan, L., Santiago, N.A., Gray, G.M., and Khosla, C. 2002. Intestinal digestive resistance of immunodominant gliadin peptides. Am. J. Physiol. Gastrointest. Liver Physiol. 283:G996-G1003.

18. Shan, L., et al. 2002. Structural basis for gluten intolerance in celiac sprue. Science. 297:2275-2279.

19. Shan, L., et al. 2005. Identification and analysis of multivalent proteolytically resistant peptides from gluten: implications for celiac sprue. J. Proteome Res. 4:1732-1741

20. Stepniak, D., et al. 2006. Highly efficient gluten degradation with a newly identified prolyl endoprotease: implications for celiac disease. Am. J. Physiol. Gastrointest. Liver Physiol. 291:G621-G629.

21. Greco, L., et al. 2002. The first large population based twin study of coeliac disease. Gut. 50:624-628.

22. Kagnoff, M.F. 2000. HLA genes in coeliac disease In Coeliac disease. S. Auricchio, L. Greco, L. Maiuri, and R. Troncone, editors. JCG Editions. Naples, Italy. 5-14.

23. Karell, K., et al. 2003. HLA types in celiac disease patients not carrying the DQA $1 * 05-\mathrm{DQB} 1 * 02$ (DQ2) heterodimer: results from the European Genetics Cluster on Celiac Disease. Hum. Immunol. 64:469-477.

24. Louka, A.S., and Sollid, L.M. 2003. HLA in coeliac disease: unravelling the complex genetics of a complex disorder. Tissue Antigens. 61:105-117.

25. Mazzarella, G., et al. 2003. An immunodominant DQ8 restricted gliadin peptide activates small intestinal immune response in in vitro cultured mucosa from HLA-DQ8 positive but not HLA-DQ8 negative coeliac patients. Gut. 52:57-62.

26. Sollid, L.M., et al. 1989. Evidence for a primary association of celiac disease to a particular HLA-DQ alpha/beta heterodimer. J. Exp. Med. 169:345-350.

27. Sollid, L.M., and Thorsby, E. 1990. The primary association of celiac disease to a given HLA-DQ alpha/ beta heterodimer explains the divergent HLA-DR associations observed in various Caucasian populations. Tissue Antigens. 36:136-137.

28. Ploski, R., Ek, J., Thorsby, E., and Sollid, L.M. 1993. On the HLA-DQ(alpha $1 * 0501$, beta $1 * 0201$ )-associated susceptibility in celiac disease: a possible gene dosage effect of DQB $1 * 0201$. Tissue Antigens. 41:173-177.

29. Louka, A.S., et al. 2002. HLA in coeliac disease families: a novel test of risk modification by the 'other' haplotype when at least one DQA $1 * 05-\mathrm{DQB} 1 * 02$ haplotype is carried. Tissue Antigens. 60:147-154.

30. Vader, W., et al. 2003. The HLA-DQ2 gene dose effect in celiac disease is directly related to the magnitude and breadth of gluten-specific T cell responses. Proc. Natl. Acad. Sci. U. S. A. 100:12390-12395.

31. Karinen, H., et al. 2006. Gene dose effect of the DQB $1 * 0201$ allele contributes to severity of coeliac disease. Scand. J. Gastroenterol. 41:191-199.

32. Makishima, H., et al. 2006. Intestinal diffuse large B-cell lymphoma associated with celiac disease: a Japanese case. Int. J. Hematol. 83:63-65.

33. Zhong, F., et al. 1996. An autosomal screen for genes that predispose to celiac disease in the western counties of Ireland. Nat. Genet. 14:329-333.

34. Greco, L., et al. 1998. Genome search in celiac disease. Am.J. Hum. Genet. 62:669-675.

35. Naluai, A.T., et al. 2001. Genome-wide linkage analysis of Scandinavian affected sib-pairs supports presence of susceptibility loci for celiac disease on chromosomes 5 and 11. Eur. J. Hum. Genet. 9:938-944.

36. Greco, L., et al. 2001. Existence of a genetic risk factor on chromosome $5 q$ in Italian coeliac disease families. Ann. Hum. Genet. 65:35-41.

37. King, A.L., et al. 2001. Coeliac disease: follow-up linkage study provides further support for existence of a susceptibility locus on chromosome 11p11. Ann. Hum. Genet. 65:377-386.

38. Liu, J., et al. 2002. Genomewide linkage analysis of celiac disease in Finnish families. Am. J. Hum. Genet. 70:51-59.

39. Neuhausen, S.L., et al. 2002. Genome-wide linkage analysis for celiac disease in North American families. Am. J. Med. Genet. 111:1-9.

40. Popat, S., et al. 2002. Genome screening of coeliac disease. J. Med. Genet. 39:328-331.

41. Percopo, S., et al. 2003. Saturation of the 5q31-q33 candidate region for coeliac disease. Ann. Hum. Genet. 67:265-268.

42. Rioux, J.D., et al. 2004. Genomewide search and association studies in a Finnish celiac disease population: identification of a novel locus and replication of the HLA and CTLA4 loci. Am.J. Med. Genet. A. 130:345-350

43. Van Belzen, M.J., et al. 2004. A genomewide screen in a four-generation Dutch family with celiac disease: evidence for linkage to chromosomes 6 and 9 . Am. J. Gastroenterol. 99:466-471.

44. Monsuur, A.J., et al. 2005. Myosin IXB variant increases the risk of celiac disease and points toward a primary intestinal barrier defect. Nat. Genet. 37:1341-1344.

45. Amundsen, S.S., et al. 2006. Association analysis of MYO9B gene polymorphisms with celiac disease in a Swedish/Norwegian cohort. Hum. Immunol. 67:341-345.

46. Hunt, K.A., et al. 2006. Lack of association of MYO9B genetic variants with coeliac disease in a British cohort. Gut. 55:969-972.

47. Lundin, K.E., et al. 1990. T lymphocyte recognition of a celiac disease-associated cis- or trans-encoded HLA-DQ alpha/beta-heterodimer. J. Immunol. 145:136-139.

48. Lundin, K.E., Scott, H., Fausa, O., Thorsby, E., and Sollid, L.M. 1994. T cells from the small intestinal mucosa of a DR4, DQ7/DR4, DQ8 celiac disease patient preferentially recognize gliadin when presented by DQ8. Hum. Immunol. 41:285-291.

49. Johansen, B.H., et al. 1996. Both alpha and beta chain polymorphisms determine the specificity of the disease-associated HLA-DQ2 molecules, with beta chain residues being most influential. Immunogenetics. 45:142-150.

50. Dieterich, W., et al. 1997. Identification of tissue transglutaminase as the autoantigen of celiac disease. Nat. Med. 3:797-801.

51. Molberg, O., et al. 1998. Tissue transglutaminase selectively modifies gliadin peptides that are recognized by gut-derived T cells in celiac disease. Nat. Med. 4:713-717.

52. Van de Wal, Y., et al. 1998. Selective deamidation by tissue transglutaminase strongly enhances gliadinspecific T cell reactivity. J. Immunol. 161:1585-1588.

53. Fleckenstein, B., et al. 2002. Gliadin T cell epitope selection by tissue transglutaminase in celiac disease. Role of enzyme specificity and $\mathrm{pH}$ influence on the transamidation versus deamidation process. J. Biol. Chem. 277:34109-34116.

54. Fleckenstein, B., et al. 2004. Molecular characterization of covalent complexes between tissue transglutaminase and gliadin peptides. J. Biol. Chem. 279:17607-17616.

55. Molberg, O., et al. 1997. Gliadin specific, HLA DQ2-restricted $T$ cells are commonly found in small intestinal biopsies from coeliac disease patients, but not from controls. Scand. J. Immunol. 46:103-109.

56. Arentz-Hansen, H., et al. 2000. The intestinal T cell response to alpha-gliadin in adult celiac disease is focused on a single deamidated glutamine targeted by tissue transglutaminase. J. Exp. Med. 191:603-612.

57. Arentz-Hansen, H., et al. 2002. Celiac lesion T cells recognize epitopes that cluster in regions of gliadins rich in proline residues. Gastroenterology. 123:803-809

58. Qiao, S.W., et al. 2004. Antigen presentation to celiac lesion-derived $\mathrm{T}$ cells of a 33-mer gliadin peptide naturally formed by gastrointestinal digestion. J. Immunol. 173:1757-1762.

59. Van de Wal, Y., et al. 1998. Small intestinal T cells of celiac disease patients recognize a natural pepsin fragment of gliadin. Proc. Natl. Acad. Sci. U. S. A. 95:10050-10054.

60. Anderson, R.P., Degano, P., Godkin, A.J., Jewell, D.P., and Hill, A.V. 2000. In vivo antigen challenge in celiac disease identifies a single transglutaminase-modified peptide as the dominant A-gliadin T-cell epitope. Nat. Med. 6:337-342.

61 . Vader, W., et al. 2002. The gluten response in children with celiac disease is directed toward multiple gliadin and glutenin peptides. Gastroenterology. 122:1729-1737.

62. Nilsen, E.M., et al. 1998. Gluten induces an intestinal cytokine response strongly dominated by interferon gamma in patients with celiac disease. Gastroenterology. 115:551-563.

63. Przemioslo, R.T., Lundin, K.E., Sollid, L.M., Nelufer, J., and Ciclitira, P.J. 1995. Histological changes in small bowel mucosa induced by gliadin sensitive $\mathrm{T}$ lymphocytes can be blocked by anti-interferon gamma antibody. Gut. 36:874-879.

64. Monteleone, I., et al. 2004. Regulation of the T helper cell type 1 transcription factor T-bet in coeliac disease mucosa. Gut. 53:1090-1095.

65. Holtmann, M.H., and Neurath, M.F. 2004. T helper cell polarisation in coeliac disease: any (T-)bet? Gut. 53:1065-1067.

66. Kim, C.Y., Quarsten, H., Bergseng, E., Khosla, C., and Sollid, L.M. 2004. Structural basis for HLA-DQ2mediated presentation of gluten epitopes in celiac disease. Proc. Natl. Acad. Sci. U. S. A. 101:4175-4179.

67. Bergseng, E., Xia, J., Kim, C.Y., Khosla, C., and Sollid, L.M. 2005. Main chain hydrogen bond interactions in the binding of proline-rich gluten peptides to the celiac disease-associated HLA-DQ2 molecule. J. Biol. Chem. 280:21791-21796

68. Qiao, S.W., et al. 2005. Refining the rules of gliadin $\mathrm{T}$ cell epitope binding to the disease-associated DQ2 molecule in celiac disease: importance of proline spacing and glutamine deamidation. J. Immunol. 175:254-261.

69. Stepniak, D., et al. 2005. T-cell recognition of HLADQ2-bound gluten peptides can be influenced by an $\mathrm{N}$-terminal proline at $\mathrm{p}-1$. Immunogenetics. 57:8-15. 
70. Tollefsen, S., et al. 2006. HLA-DQ2 and -DQ8 signatures of gluten $\mathrm{T}$ cell epitopes in celiac disease. J. Clin. Invest. 116:2226-2236. doi:10.1172/JCI27620.

71. Vader, L.W., et al. 2002. Specificity of tissue transglutaminase explains cereal toxicity in celiac disease. J. Exp. Med. 195:643-649.

72. Anderson, R.P., et al. 2005. T cells in peripheral blood after gluten challenge in coeliac disease. Gut. 54:1217-1223.

73. Bardella, M.T., Marino, R., and Meroni, P.L. 1999. Celiac disease during interferon treatment. Ann. Intern. Med. 131:157-158.

74. Cammarota, G., Cuoco, L., Cianci, R., Pandolfi, F., and Gasbarrini, G. 2000. Onset of coeliac disease during treatment with interferon for chronic hepatitis C. Lancet. 356:1494-1495.

75. Monteleone, G., Pender, S.L., Wathen, N.C., and MacDonald, T.T. 2001. Interferon-alpha drives T cell-mediated immunopathology in the intestine. Eur. J. Immunol. 31:2247-2255.

76. Jabri, B., et al. 2000. Selective expansion of intraepithelial lymphocytes expressing the HLA-E-specific natural killer receptor CD94 in celiac disease. Gastroenterology. 118:867-879.

77. Hue, S., et al. 2004. A direct role for NKG2D/MICA interaction in villous atrophy during celiac disease. Immunity. 21:367-377.

78. Meresse, B., et al. 2004. Coordinated induction by IL15 of a TCR-independent NKG2D signaling pathway converts CTL into lymphokine-activated killer cells in celiac disease. Immunity. 21:357-366.

79. Meresse, B., et al. 2006. Reprogramming of CTLs into natural killer-like cells in celiac disease. J. Exp. Med. 203:1343-1355.

80. Cellier, C., etal. 1998. Abnormal intestinal intraepithelial lymphocytes in refractory sprue. Gastroenterology. 114:471-481.
81. Maiuri, L., et al. 2000. Interleukin 15 mediates epithelial changes in celiac disease. Gastroenterology. 119:996-1006.

82. Maiuri, L., et al. 2001. IL-15 drives the specific migration of CD94+ and TCR-gammadelta+ intraepithelial lymphocytes in organ cultures of treated celiac patients. Am. J. Gastroenterol. 96:150-156.

83. Mention, J.J., et al. 2003. Interleukin 15: a key to disrupted intraepithelial lymphocyte homeostasis and lymphomagenesis in celiac disease. Gastroenterology. 125:730-745.

84. Maiuri, L., et al. 2003. Association between innate response to gliadin and activation of pathogenic $\mathrm{T}$ cells in coeliac disease. Lancet. 362:30-37.

85. Rescigno, M., et al. 2001. Dendritic cells express tight junction proteins and penetrate gut epithelial monolayers to sample bacteria. Nat. Immunol. 2:361-367.

86. Pender, S.L., et al. 1997. A major role for matrix metalloproteinases in $\mathrm{T}$ cell injury in the gut. J. Immunol. 158:1582-1590.

87. Daum, S., et al. 1999. Increased expression of mRNA for matrix metalloproteinases- 1 and -3 and tissue inhibitor of metalloproteinases- 1 in intestinal biopsy specimens from patients with coeliac disease. Gut. 44:17-25.

88. Franco, A., et al. 1994. Peripheral T cell response to A-gliadin in celiac disease: differential processing and presentation capacities of Epstein-Barrtransformed B cells and fibroblasts. Clin. Immunol. Immunopathol. 71:75-81.

89. Gjertsen, H.A., Sollid, L.M., Ek, J., Thorsby, E., and Lundin, K.E. 1994. T cells from the peripheral blood of coeliac disease patients recognize gluten antigens when presented by HLA-DR, -DQ, or -DP molecules. Scand. J. Immunol. 39:567-574.

90. Molberg, O., et al. 1998. HLA restriction patterns of gliadin- and astrovirus-specific CD4+ T cells isolated in parallel from the small intestine of celiac disease patients. Tissue Antigens. 52:407-415.

91. Xia, J., Siegel, M., Bergseng, E., Sollid, L.M., and Khosla, C. 2006. Inhibition of HLA-DQ2-mediated antigen presentation by analogues of a high affinity 33-residue peptide from alpha2-gliadin. J. Am. Chem. Soc. 128:1859-1867.

92. Ciclitira, P.J., Hunter, J.O., and Lennox, E.S. 1980. Clinical testing of bread made from nullisomic $6 \mathrm{~A}$ wheats in coeliac patients. Lancet. 2:234-236.

93. Molberg, O., et al. 2005. Mapping of gluten T-cell epitopes in the bread wheat ancestors: implications for celiac disease. Gastroenterology. 128:393-401.

94. Spaenij-Dekking, L., et al. 2005. Natural variation in toxicity of wheat: potential for selection of nontoxic varieties for celiac disease patients. Gastroenterology. 129:797-806.

95. Khosla, C., Gray, G.M., and Sollid, L.M. 2005. Putative efficacy and dosage of prolyl endopeptidase for digesting and detoxifying gliadin peptides. Gastroenterology. 129:1362-1363; author reply 1363.

96. Shan, L., Marti, T., Sollid, L.M., Gray, G.M., and Khosla, C. 2004. Comparative biochemical analysis of three bacterial prolyl endopeptidases: implications for coeliac sprue. Biochem. J. 383:311-318.

97. Piper, J.L., Gray, G.M., and Khosla, C. 2004. Effect of prolyl endopeptidase on digestive-resistant gliadin peptides in vivo. J. Pharmacol. Exp. Ther. 311:213-219.

98. Stepniak, D., et al. 2006. Highly efficient gluten degradation with a newly identified prolyl endoprotease: implications for celiac disease. Am.J. Physiol. Gastrointest. Liver Physiol. 291:G621-G629.

99. Siegel, M., et al. 2006. Rational design of combination enzyme therapy for celiac sprue. Chem. Biol. 13:649-658. 